\title{
Vanishing aneurysms in Behcet's disease - A case series
}

\author{
Taruna Yadav $^{1}$, Suvinay Saxena ${ }^{1}$, Naveen Dutt ${ }^{2}$, Pawan Garg ${ }^{1}$, Pushpinder Khera ${ }^{1}$ \\ ${ }^{1}$ Department of Diagnostic and Interventional Radiology; ${ }^{2}$ Department of Pulmonary Medicine, All India Institute of \\ Medical Sciences, Jodhpur, India
}

\begin{abstract}
Behçet's disease is a systemic vasculitis with a wide spectrum of manifestations. Although pulmonary involvement is typical, the presence of pulmonary arterial aneurysms is associated with a high incidence of mortality and morbidity. We report two cases of Behçet's disease with pulmonary arterial involvement who presented with hemoptysis, showed significant treatment response, thus, highlighting the importance of early diagnosis and therapeutic management.
\end{abstract}

Correspondence: Pawan Garg, Associate Professor, Department of Diagnostic and Interventional Radiology, All India Institute of Medical Sciences, Jodhpur, Rajasthan 342005, India.

Mobile: +91.8003995327 .

E-mail: drgargpawan@gmail.com

Author contributions: TY, conceptualized, edited the manuscript and reviewed the literature. SS, collected the data and prepared first draft of manuscript; ND, analysed the data and reviewed the literature; PG, edited, reviewed the manuscript and is the article guarantor; PK, reviewed the final draft.

Conflicts of interest: The authors declare that they have no competing interests, and all authors confirm accuracy.

Ethics approval and consent to participate: No ethical committee approval was required for this case report by the Department, because this article does not contain any studies with human participants or animals.

Consent for publication: Informed consent was obtained from the patients.

Keywords: Behçet's disease; pulmonary arterial aneurysm; thrombosis; vasculitis; computed tomography pulmonary angiography.

Received for publication: 11 June 2020.

Accepted for publication: 17 November 2020.

${ }^{\circ}$ Copyright: the Author(s), 2021

Licensee PAGEPress, Italy

Monaldi Archives for Chest Disease 2021; $91: 1451$

doi: 10.4081/monaldi.2021.1451

This article is distributed under the terms of the Creative Commons Attribution Noncommercial License (by-nc 4.0) which permits any noncommercial use, distribution, and reproduction in any medium, provided the original author(s) and source are credited.

\section{Introduction}

Behçet's disease (BD) is a multisystemic chronic relapsing inflammatory disorder with uncertain etiology. The classical triad was first described by Turkish dermatologist, Hulusi Behçet in 1937. It consists of the triad of recurrent oral aphthous ulcers, genital ulcers, and uveitis. It can affect almost all systems including the ocular, gastrointestinal, central nervous system, cardio-thoracic, and joints [1].

The diagnosis of $\mathrm{BD}$ is made on the basis of diagnostic criteria established by the International Criteria for Behçet's disease (ICBD). According to ICBD, scoring of $\geq 4$ points classifies as Behçet's disease. Ocular lesions, genital ulcers, and oral aphthosis carry 2 points each. Skin lesions, neurological involvement, vascular manifestations, and Positive pathergy test carry 1 point each. However, the primary scoring system does not include pathergy testing and is considered optional [2].

Approximately $25-30 \%$ of $\mathrm{BD}$ cases demonstrate vascular involvement, with pulmonary involvement being uncommon with incidence rates ranging from $1-10 \%$. The pulmonary manifestations of BD have been regrouped as - Aneurysms of the pulmonary artery, pulmonary parenchymal changes, and miscellaneous group -consisting of pulmonary artery occlusions, pleural effusion, and pulmonary obstructive airway disease. Pulmonary arterial aneurysms are not uncommon and are stipulated to be a result of inflammatory endarteritis causing destruction of media resulting in an aneurysm [3-5].

We report two cases to display their unusual presentation and excellent response to medical management after timely diagnosis.

\section{Case \#1}

An 18-year-old non-smoker male patient presented to the emergency room with an episode of massive hemoptysis. He had no history of cough, breathlessness, chest pain. No history of drug intake, allergy, substance abuse, and familial illnesses was present. He had a history of attending the ophthalmology clinic for the last 3 months. He had a history of recurrent oral and genital ulcers since childhood. On examination, he had mild tachycardia (HR$105)$, tachypnoea. His oxygen saturation was $96 \%$ on room air. He did not have any oral or genital ulcers at present. His laboratory workup revealed normal hemogram. ESR was elevated (69 $\mathrm{mm} / \mathrm{h}$ ). Ophthalmological examination revealed bilateral vitritis and retinitis. His autoimmune profile (rheumatoid factor, antinuclear antibodies) and viral serology were all negative. Pathergy test skin hypersensitivity to needle prick was negative after $48 \mathrm{~h}$. Chest radiograph showed few well-defined opacities along the right lower perihilar and left hilar region. Few small round opaci- 
ties were also seen along bilateral upper parahilar regions (Figure $1 \mathrm{a}, \mathrm{b})$.

The computed tomography pulmonary angiography (CTPA) demonstrated multiple variable-sized fusiform and saccular pulmonary arterial aneurysms involving almost all lobes of bilateral lungs. Aneurysms had irregular and enhancing thickened walls with few of them showing partial eccentric thrombi. Branches of the left lower lobe pulmonary artery were thrombosed (Figure 2 ac). HLA B5 was negative. In addition, the workup for a hypercoagulable state did not reveal any abnormality. Based on the clinical and radiological evidence, a diagnosis of Behçet's disease was made. He was treated with immunosuppressants (azathioprine and pulse steroid-methylprednisolone therapy). He responded well to treatment with no recurrence of hemoptysis. Follow up CTPA after 1 year showed near-total resolution of pulmonary artery aneurysms (Figure 2 d,e,f).

\section{Case \#2}

A 24-year-old male patient visited the hospital with complaints of intermittent hemoptysis on and off for the last 1 year, approximately $15-20 \mathrm{ml} /$ day, and the last episode was 4 days back. He had no history of other respiratory symptoms. Past history of oral ulcers was present. He had an episode of deep venous thrombosis in both lower limbs, 2 years back, and history of inferior mesenteric vein thrombosis, a year back. On examination, he had normal vitals. He had an erythematous nodule (erythema-nodosum-like lesion) on the right forearm which was biopsied and histopathology revealed features consistent with vasculitis. His autoimmune profile (rheumatoid factor, antinuclear antibodies), HLA B5, pathergy, and Mantoux tests were negative. There was no uveitis or eye involvement on ophthalmological examination with normal neurological examination. CTPA revealed multiple bilateral pul- monary arterial aneurysms. Few areas of partial and complete thrombosis were seen in some of these pulmonary arterial aneurysms and branches (Figure $3 \mathrm{a}, \mathrm{b}, \mathrm{c}$ ).

The diagnosis of BD was made and the patient was started on immunosuppressants (Azathioprine and pulsed steroid-methylprednisolone therapy). On subsequent follow-up CTPA scan after 2 months, partial resolution of pulmonary arterial aneurysms was observed with the clearing of eccentric thrombi in some of them (Figure 3 d,e,f). The patient is still continuing treatment with immunosuppressants.

\section{Discussion}

Behçet's disease is a vasculitis that involves small, large arteries and veins. Venous involvement being more common. Arterial affliction results in the formation of aneurysms and thrombosis. It is the most common cause of pulmonary artery aneurysms which are one of the causes of mortality in these patients. It predominantly affects young males as seen in our cases. The most common presenting symptom is hemoptysis which can occur due to pulmonary arterial aneurysmal rupture with bronchial communication.

The etiopathogenesis of $\mathrm{BD}$ is unspecified, however various associations with human leukocyte antigen HLA-B51, genetic mutations including factor $\mathrm{V}$ Leiden, and multiple other discrete alleles of HLA have been identified [6-8]. Inflammation of the vasa-vasorum of the tunica media layer results in the destruction of elastic fibers of media with consequent development of vessel lumen dilatation and subsequent aneurysm formation [9].

Hughes-Stovin syndrome (HSS) is a close differential with overlapping radiological and histopathological features but with the absence of oral or genital ulcers [4]. The clinical presentation of our patients was consistent with BD.

Vascular complications of BD including pulmonary arterial

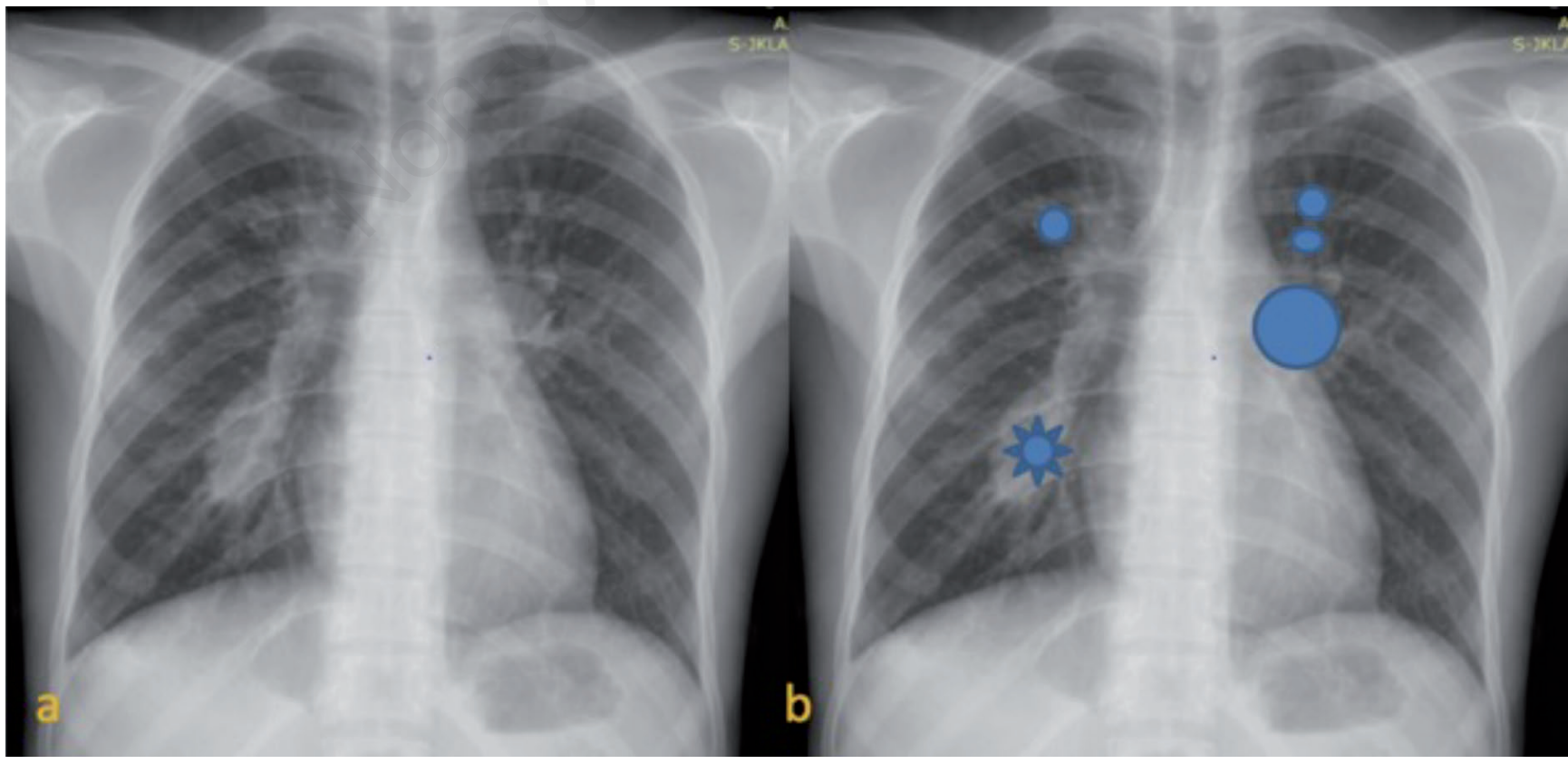

Figure 1. Frontal chest radiograph (a, original; b, marked) shows a fusiform opacity in right lower perihilar and another round opacity along the left hilar region. Few small suspicious round parenchymal opacities are also seen in bilateral upper perihilar regions. 


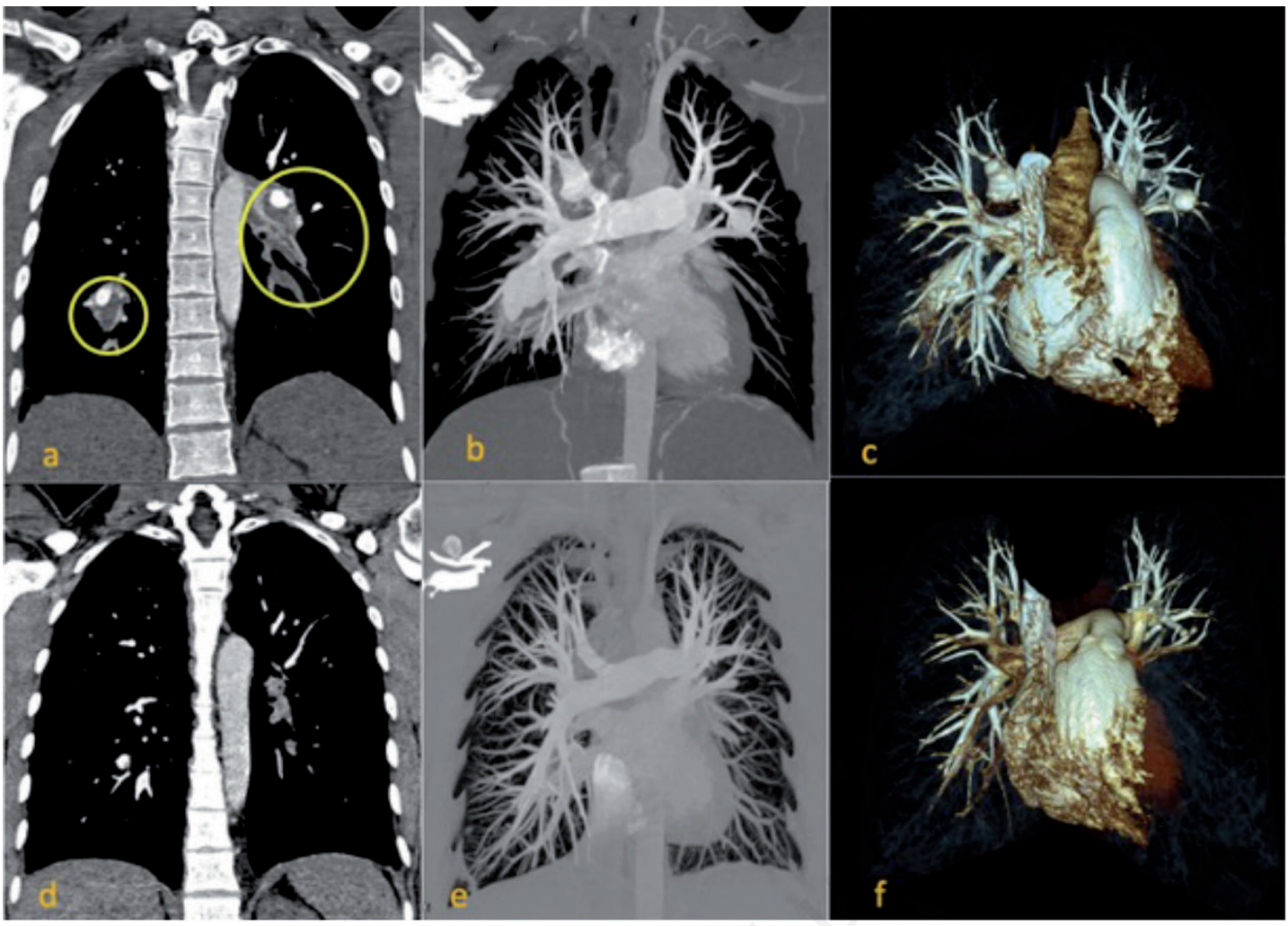

Figure 2. Pre-treatment (a,b,c) CT pulmonary angiography (CTPA) coronal (a), coronal maximum intensity projection (MIP) (b) and volume-rendered (VR) (c) images reveal multiple aneurysms involving bilateral pulmonary arteries with irregular enhancing mural thickening and eccentric thrombi (circles in a). Post-treatment (d,e,f) CTPA after 1 year shows near-total resolution of pulmonary artery aneurysms. The left descending pulmonary artery shows chronic thrombosis with no recanalization.

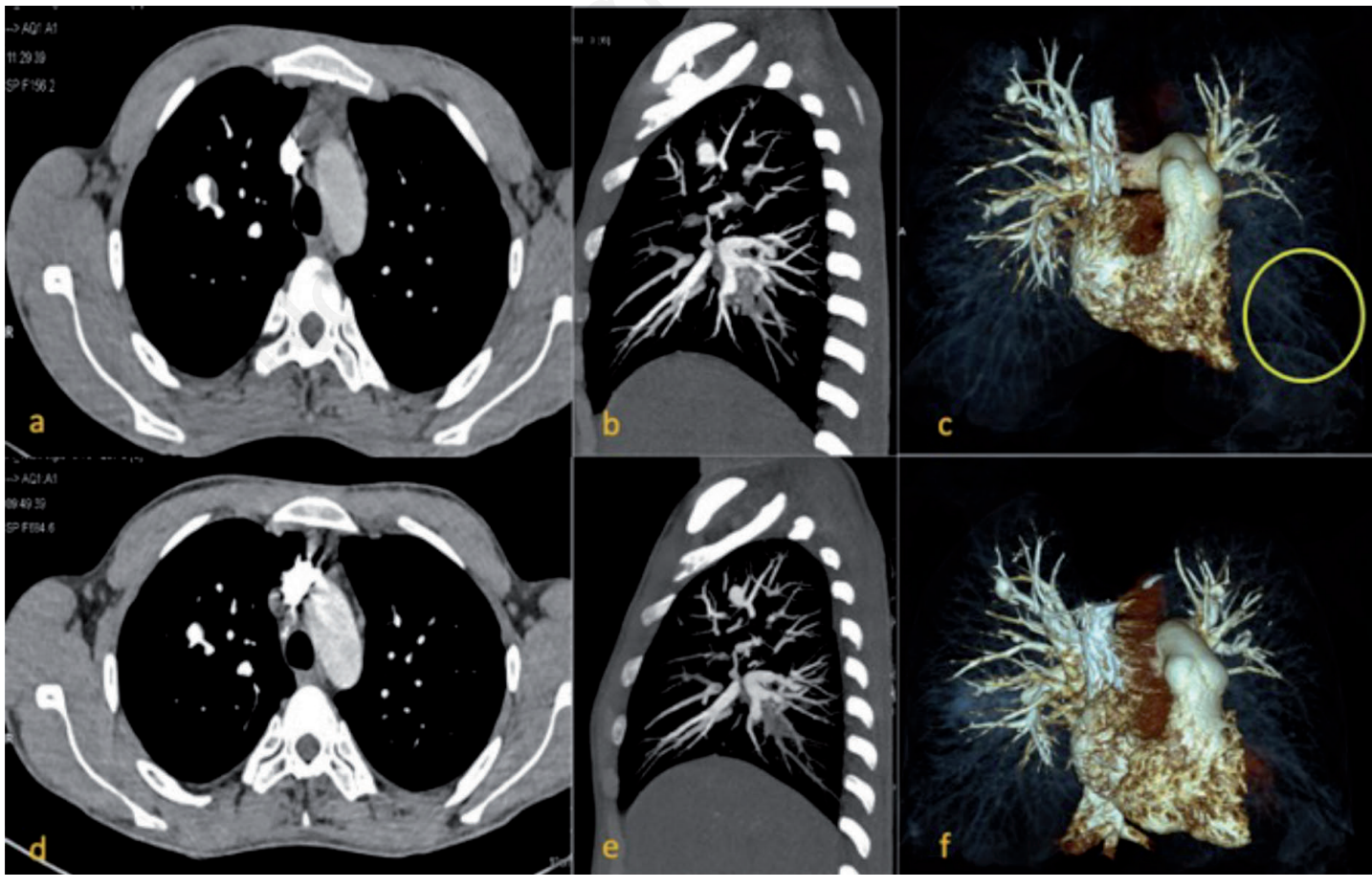

Figure 3. Pre-treatment (a,b,c) CTPA axial (a), right sagittal MIP (b) and VR (c) images show multiple variable sized fusiform pulmonary artery aneurysms. Complete thrombosis of the left descending pulmonary artery (circle) resulted in its non-visualisation on VRT images. Post-treatment (d,e,f) CTPA after 2 months reveals mild reduction in the size of aneurysms with resolution of mural thrombi in these aneurysms. 
aneurysms respond well to treatment with immunosuppressants. In our first case, pulmonary arterial aneurysms showed near-complete resolution after treatment. Good response was also seen in the second case with decrease in thrombus load and decrease in the size of aneurysms within a short time span. Prognosis depends upon the age of presentation, duration of symptoms, and early treatment initiation [10].

To conclude, the computed tomography pulmonary angiography (CTPA) plays a pivotal role in the diagnosis of vascular as well as lung parenchymal manifestations, aids in guiding the management, and is an indispensable tool for follow up of such patients. Early diagnosis and treatment of Behçet's disease is critical for the prevention of catastrophic and potentially fatal complication of pulmonary arterial aneurysmal rupture.

\section{References}

1. Behçet H. [Uber rezidivierende aphthose, durch ein virus verursachte Geschwure am Mund, am Auge und an den Genitalien].[Article in German]. Dermatol Woschensch. 1937;105:1152-7.

2. International Team for the Revision of the International Criteria for Behçet's Disease (ITR-ICBD). The International Criteria for Behçet's Disease (ICBD): a collaborative study of 27 countries on the sensitivity and specificity of the new crite- ria. J Eur Acad Dermatol Venereol 2014;28:338-47.

3. Nabih M, Naguib S, El Menyawi M, Ismail I. Pulmonary manifestations in a group of patients with Behçet's disease. Egypt J Intern Med 2015;27:8.

4. Hiller N, Lieberman S, Chajek-Shaul T, et al. Thoracic manifestations of Behçet disease at CT. RadioGraphics 2004;24:801-8.

5. Ceylan N, Bayraktaroglu S, Erturk SM, Savas R, Alper H. Pulmonary and vascular manifestations of Behçet disease: Imaging findings. Am J Roentgenol 2010;194:W158-64.

6. Kang EH1, Kim JY, Takeuchi F, et al. Associations between the HLA-A polymorphism and the clinical manifestations of Behcets disease. Arthritis Res Ther 2011;13:R49.

7. Agha A, Bella AM, Assiri AH, Al-Hakami M. Can Behcet's disease related pulmonary arterial aneurysms be completely resolved? Open Rheumatol J 2011;5:88-90.

8. de Albuquerque Botura C, Bersani-Amado LE, ZacariasJúnior AR, et al. Behçet's disease: Pulmonary aneurysm resolution with oral therapy and HLA B72 allele association. Clin Med Res 2018;16:37-40.

9. Aktogu S, Erer OF, Urpek G, et al. Multiple pulmonary arterial aneurysms in Behcet's disease: clinical and radiologic remission after cyclophosphamide and corticosteroid therapy. Respiration 2002;69:178-81.

10. Hamuryudan V, Yurdakul S, Moral F, et al. Pulmonary arterial aneurysms in Behçet's syndrome: a report of 24 cases. Br J Rheumatol 1994;33:48-5. 